\title{
Tuberculose e indicadores socioeconômicos: revisão sistemática da literatura
}

\author{
Alexandre San Pedro ${ }^{1}$ e Rosely Magalhães de Oliveira ${ }^{1}$
}

Como citar San Pedro A, Oliveira RM. Tuberculose e indicadores socioeconômicos: revisão sistemática da literatura. Rev Panam Salud Publica. 2013;33(4):294-301.

RESUMO Objetivo. Analisar a literatura para verificar a existência de associação entre fatores socioeconômicos (individuais e coletivos) e a ocorrência de tuberculose.

Métodos. Realizou-se uma revisão sistemática da literatura mediante busca nas bases de dados SciELO, LILACS, Medline e Scopus utilizando os termos "pobreza, indicadores sociais, fatores socioeconômicos" e "tuberculose" (em português, inglês e espanhol). Os estudos de nível individual foram classificados segundo o desenho de estudo e a variável dependente; os estudos ecológicos, quanto aos diferentes níveis de agregação espacial dos dados e à variável dependente. Para cada artigo foram registradas informações concernentes ao título do estudo, país de origem, ano de desenvolvimento, autor, idioma, objetivos, nível de agregação espacial dos dados e indicadores utilizados na análise.

Resultados. Para estudos de nível individual, observou-se associação estatística direta entre tuberculose e alcoolismo, coinfecção com HIV, baixa escolaridade, estado civil, baixo rendimento monetário, carência alimentar, imigração e contato prévio com pacientes de tuberculose. Em nível coletivo, uma associação indireta foi verificada com variáveis referentes ao produto interno bruto per capita, indice de desenvolvimento humano e acesso a saneamento básico em nível de países. Indicadores relativos ao número médio de pessoas por cômodo, densidade de pobres, escolaridade, declínio da renda familiar e domicílios com ajuda monetária governamental associaram-se diretamente a tuberculose em diferentes níveis de agregação espacial.

Conclusões. Os estudos analisados apontam para a persistência da relação entre indicadores socioeconômicos e a produção da tuberculose tanto em nível individual quanto coletivo. A associação entre tuberculose e indicadores socioeconômicos parece ser influenciada tanto pelo nível de agregação espacial quanto pelas características particulares das áreas geográficas.

Palavras-chave Tuberculose; fatores socioeconômicos; pobreza; revisão.

A tuberculose é considerada uma das mais antigas doenças infecciosas da humanidade e, embora passível de um efetivo tratamento, permanece na atualidade como um importante problema de

\footnotetext{
Fundação Oswaldo Cruz (FIOCRUZ), Escola Nacional de Saúde Pública Sergio Arouca (ENSP), Departamento de Endemias Samuel Pessoa, Rio de Janeiro (RJ), Brasil. Correspondência: Alexandre San Pedro, alexsan@ensp.fiocruz.br
}

saúde pública mundial, em virtude da ampla dispersão geográfica, emergência de casos multirresistentes e coinfecção com HIV. Estima-se que, no ano de 2010, ocorreram no mundo aproximadamente 8,8 milhões de casos novos, 1,1 milhão de óbitos entre indivíduos não portadores de HIV e 400000 óbitos entre pessoas soropositivas para HIV $(1,2)$.

A influência marcante e persistente das condições de vida no processo de trans- missão da tuberculose vem ressaltando um profundo quadro de desigualdades socioeconômicas que resultam em iniquidades sociais em saúde (3). Estudos recentes sugerem que as modificações nas estimativas nacionais de incidência da tuberculose estão mais associadas às mudanças nos índices socioeconômicos e estado geral de saúde da população do que ao desempenho de programas de controle desse agravo $(4,5)$. 
Diversas propostas metodológicas vêm sendo consideradas para operacionalizar as "condições de vida" como uma categoria de pesquisa. Tais propostas buscam uma compreensão dos diferentes riscos individuais e coletivos de adoecimento e morte relacionados ao processo de transmissão da doença. Nesse sentido, destacam-se os estudos de associação entre fatores das esferas biológica e social e sua influência na produção e reprodução da tuberculose (6-10). No entanto, os estudos que buscam associar a ocorrência de tuberculose com indicadores socioeconômicos (representantes das condições de vida) nem sempre encontram resultados concordantes. Tal divergência pode estar relacionada ao nível de agregação territorial dos dados, assim como a características particulares inerentes às populações em estudo.

O objetivo deste trabalho foi realizar uma revisão sistemática da literatura para investigar a existência de associação entre fatores socioeconômicos (individuais e coletivos) e a ocorrência de tuberculose.

\section{MATERIAIS E MÉTODOS}

Os termos de busca utilizados nesta revisão sistemática foram obtidos através de consulta aos Descritores em Ciências da Saúde (decs.bvs.br). Foi utilizada na busca dos trabalhos a combinação dos descritores "pobreza, indicadores sociais, fatores socioeconômicos" e "tuberculose" (em espanhol, pobreza, indicadores sociales, fatores socioeconómicos; e em inglês poverty, social indicators, socioeconomic factors). Na pesquisa bibliográfica foram utilizadas as bases SciELO (www. scielo.org), LILACS (bases. bireme.br), Medline (www.ncbi.nlm.nih.gov/pubmed) e Scopus (www.scopus.com/periodicos.capes.gov.br/home.url).

Foram inicialmente selecionados, através dos descritores, trabalhos publicados entre 1990 e 2011. A partir daí, foram selecionados artigos originais e comunicações breves (de acesso livre ou disponibilizados pelo Portal de Periódicos da Coordenação de Aperfeiçoamento de Pessoal de Nível Superior, CAPES, no Brasil), assim como teses e dissertações. Foram critérios de inclusão a presença de resumos (em português, inglês ou espanhol), assim como a descrição de abordagem quantitativa referente à análise de associação estatística entre tuberculose e fatores socioeconômicos. Foram excluí- dos os estudos de abordagem qualitativa e os de cunho quantitativo que apresentaram em seu resumo apenas a descrição de medidas de frequência. Foram ainda excluídos os capítulos de livro, resumos de eventos, relatos de caso, editoriais, revisões sistemáticas, meta-análises e artigos de opinião.

Ambos os autores participaram de forma independente da avaliação de elegibilidade e posterior análise das publicações, sendo os desacordos resolvidos por consenso. Para a extração dos dados, foi construído um formulário especifico, onde foram registradas informações concernentes ao título do estudo, país de origem, ano de desenvolvimento, autor, idioma, objetivo, variável dependente, nível de agregação espacial dos dados e indicadores socioeconômicos utilizados na análise.

Os trabalhos selecionados foram separados segundo tipo de estudo (ecológico ou individuado, no qual a unidade de análise é o indivíduo). Por sua vez, os trabalhos de nível individuado foram classificados de acordo com o desenho de estudo (caso-controle, coorte e inquéritos) e variável dependente (mortalidade, incidência, prevalência, coinfecção e abandono). Por outro lado, os ecológi$\cos$ foram classificados quanto à variável dependente e os diferentes níveis de agregação espacial dos dados (regiões continentais/conjunto de países, país, província/estados, município, distritos, bairros e setores censitários).

As publicações selecionadas para revisão foram avaliadas quanto a critérios de qualidade, sendo considerados os artigos julgados pelo sistema de revisão por pares. Para os estudos observacionais foi utilizada como referência a lista proposta pelo STROBE Statement (11). Para os estudos ecológicos foi utilizada uma adaptação da referida lista STROBE considerando-se a adequação dos modelos, magnitude e significância da associação.

\section{RESULTADOS}

A partir dos critérios estabelecidos para revisão bibliográfica, foram selecionados ao todo 36 trabalhos (figura 1), sendo 25 referentes ao nível ecológico e 11 ao nível individuado.

\section{Estudos de nível individuado}

Dos 11 trabalhos que analisaram os fatores socioeconômicos associados à tu-
FIGURA 1. Fluxograma de seleção de estudos sobre associação entre fatores socioeconômicos e tuberculose para inclusão na revisão bibliográfica, 1990 a 2011

Publicações potencialmente elegíveis identificadas nas bases bibliográficas através dos descritores: 432

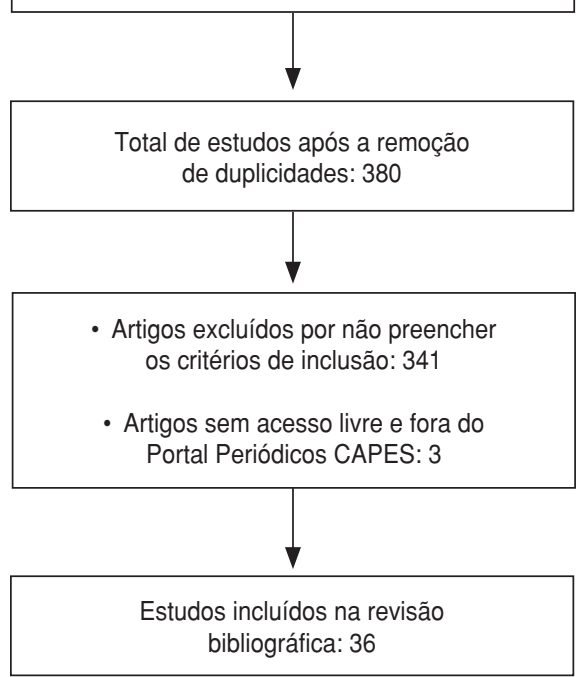

berculose em nível do indivíduo, quatro foram estudos do tipo caso-controle, três foram de coorte e quatro foram inquéritos (tabela 1).

Em relação a casos incidentes de tuberculose, os estudos com delineamento caso-controle (realizados no Brasil e na Estônia) apontaram associação positiva para as seguintes variáveis: sexo (masculino), faixa etária (30 a 54 anos), analfabetismo, imigração, posse de poucos bens de consumo, baixa renda ou rendimento não fixo, histórico prisional, alcoolismo, estado civil (separado, viúvo ou solteiro), carência alimentar e contato prévio com paciente de tuberculose $(6,12)$.

Em relação à prevalência de casos, foi verificada associação direta para as variáveis coinfecção com HIV, imigração, contato prévio com paciente de tuberculose e disponibilidade de alimentos (<2 refeições diárias) (13).

Quanto aos fatores associados ao óbito pela doença, um estudo apontou associação positiva para indivíduos com idade superior a 50 anos e alcoolismo como doença associada, enquanto que estar empregado foi um fator de proteção (7).

Entre os estudos de coorte, um apontou para associação positiva entre óbito por tuberculose, idade superior a 50 anos, desemprego e coinfecção com HIV (14). Em relação ao abandono de tratamento, foi verificada associação positiva 
TABELA 1. Estudos de nível individuado sobre associação entre fatores socioeconômicos e tuberculose

\begin{tabular}{|c|c|c|c|c|c|c|}
\hline Referência & País & Ano & Tipo de estudo & Amostra & $\begin{array}{c}\text { Variável } \\
\text { dependente }\end{array}$ & Indicadores associados ${ }^{a}$ \\
\hline $\begin{array}{l}\text { Tekkel et al. } \\
\qquad(12)\end{array}$ & Estônia & 2002 & $\begin{array}{l}\text { Caso-controle } \\
\text { (hospitalar) }\end{array}$ & $\begin{array}{l}248 \text { casos e } \\
248 \text { controles }\end{array}$ & Incidência & $\begin{array}{l}\text { Rendimento monetário não fixo }(\mathrm{AOR}=12,3 ; \mathrm{IC}=3,1-47,3) \text {, histórico } \\
\text { prisional }(\mathrm{AOR}=12,4 ; \mathrm{IC}=3,5-43,2) \text {, alcoolismo }(\mathrm{AOR}=13,6 ; \\
\text { IC =4,6-40,1), estado civil separado }(\mathrm{AOR}=2,7 ; \mathrm{IC}=1,4-5,2), \\
\text { viúvo }(\mathrm{AOR}=3,5 ; \mathrm{IC}=1,04-12,3) \text {, carência alimentar }(\mathrm{AOR}=5,2 ; \\
\text { IC =1,2-24,2), contato prévio com paciente de tuberculose } \\
(\mathrm{AOR}=7,7 ; \mathrm{IC}=3,2-18,7) .\end{array}$ \\
\hline $\begin{array}{l}\text { Cheng et al. } \\
\text { (19) }\end{array}$ & China & 2005 & $\begin{array}{l}\text { Inquérito } \\
\text { (ambulatorial) }\end{array}$ & 190 & $\begin{array}{l}\text { Atraso no } \\
\text { diagnóstico }\end{array}$ & $\begin{array}{l}\text { Faixa etária }(<30 \text { anos })(R R=0,4 ; I C=0,2-0,7) \text { e analfabetismo } \\
\quad(R R=1,9 ; I C=1,1-3,1) .\end{array}$ \\
\hline $\begin{array}{l}\text { Pelaquin et al. } \\
\text { (7) }\end{array}$ & Brasil & 2007 & $\begin{array}{l}\text { Caso-controle } \\
\text { (hospitalar) }\end{array}$ & $\begin{array}{l}48 \text { casos e } \\
96 \text { controles }\end{array}$ & Mortalidade & $\begin{array}{l}\text { Idade superior a } 50 \text { anos }(\mathrm{OR}=8,9 ; \mathrm{IC}=2,4-31,9) \text {, alcoolismo } \\
\quad(\mathrm{OR}=25,0 ; \mathrm{IC}=7,0-88,8), \text { possuir emprego }(\mathrm{OR}=0,23 \\
\mathrm{IC}=0,6-0,7)\end{array}$ \\
\hline $\begin{array}{l}\text { Albuquerque } \\
\text { et al. (14) }\end{array}$ & Brasil & 2007 & $\begin{array}{c}\text { Coorte } \\
\text { (ambulatorial) }\end{array}$ & 1237 & $\begin{array}{l}\text { Abandono, falha } \\
\text { terapêutica } \\
\text { e óbitos }\end{array}$ & 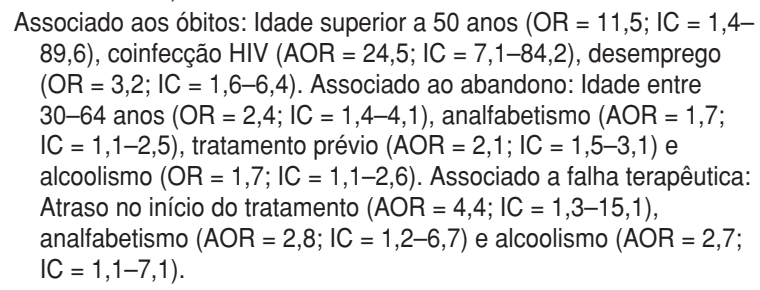 \\
\hline $\begin{array}{l}\text { Muniyandi et al. } \\
\qquad(18)\end{array}$ & Índia & 2007 & $\begin{array}{l}\text { Inquérito } \\
\text { (populacional) }\end{array}$ & 151 & Prevalência & $\begin{array}{l}\text { Renda inferior a um dólar por dia }(\mathrm{RR}=1,6 ; P<0,01) \text {, não ter } \\
\text { posse da terra (RR }=3,3 ; P<0,01) \text { e viver em habitações com } \\
\text { precariedade estrutural }(\mathrm{RR}=2,5 ; P<0,01) \text {. }\end{array}$ \\
\hline $\begin{array}{l}\text { Nájera-Ortiz } \\
\text { et al. (20) }\end{array}$ & México & 2008 & $\begin{array}{l}\text { Inquérito } \\
\text { (ambulatorial) }\end{array}$ & 295 & Mortalidade & $\begin{array}{l}\text { Menos de } 3 \text { anos de estudo }(O R=3,3 ; I C=1,1-9,6) \text {, abandono de } \\
\text { tratamento }(O R=11,5 ; I C=5,3-24,8) \text {, não ter recebido tratamento } \\
\text { diretamente observado (DOT) }(O R=1,2 ; I C=1,1-1,3) \text {. }\end{array}$ \\
\hline $\begin{array}{l}\text { Kittikraisak et } \\
\text { al. (15) }\end{array}$ & Tailândia & 2009 & $\begin{array}{c}\text { Coorte } \\
\text { (hospitalar) }\end{array}$ & 554 & $\begin{array}{l}\text { Abandono de } \\
\text { tratamento }\end{array}$ & $\begin{array}{l}\text { Histórico carcerário }(A O R=2,0 ; I C=1,1-3,2) \text {, sintomas clínicos } \\
\text { desfavoráveis após a quarta semana de tratamento }(A O R=3,4 \text {; } \\
I C=1,4-8,0) \text {, fumante }(A O R=2,3 ; I C=1,3-4,1) \text {. }\end{array}$ \\
\hline $\begin{array}{l}\text { Ximenes et al. } \\
(6)\end{array}$ & Brasil & 2009 & $\begin{array}{l}\text { Caso-controle } \\
\text { (populacional) }\end{array}$ & $\begin{array}{l}1452 \text { casos e } \\
5808 \text { controles }\end{array}$ & Incidência & $\begin{array}{l}\text { Sexo masculino }(O R=2,2 ; I C=1,9-2,5) \text {, faixa etária de } 30 \text { a } 54 \text { anos } \\
(O R=3,7 ; I C=2,9-4,8), \text { analfabetismo }(O R=1,3 ; I C=1,1-1,6), \\
\text { migração }(O R=1,3 ; I C=1,1-1,5), \text { posse de poucos bens de } \\
\text { consumo }(O R=5,5 ; I C=3,5-7,6) .\end{array}$ \\
\hline $\begin{array}{l}\text { Boccia et al. } \\
\qquad(13)\end{array}$ & Zâmbia & 2011 & $\begin{array}{l}\text { Caso-controle } \\
\text { (populacional) }\end{array}$ & $\begin{array}{l}52 \text { casos e } \\
318 \text { controles }\end{array}$ & Prevalência & $\begin{array}{l}\text { Carência alimentar }(<2 \text { refeições diárias) }(\mathrm{AOR}=3,1 ; \mathrm{IC}=1,1-8,7) \text {, } \\
\text { coinfeccão HIV }(\mathrm{AOR}=3,1 ; \mathrm{IC}=1,7-5,8) \text {, migração }(\mathrm{AOR}=5,2 ; \\
\mathrm{IC}=2,7-10,2) \text {, contato nos } 12 \text { últimos meses com paciente de } \\
\text { tuberculose }(\mathrm{AOR}=2,8 ; \mathrm{IC}=1,3-5,6) .\end{array}$ \\
\hline $\begin{array}{l}\text { Belo et al. } \\
\quad(16)\end{array}$ & Brasil & 2011 & $\begin{array}{c}\text { Coorte } \\
\text { (hospitalar) }\end{array}$ & 460 & $\begin{array}{l}\text { Desfecho negativo } \\
\text { (abandono, falha } \\
\text { terapêutica e óbitos) }\end{array}$ & $\begin{array}{l}\text { Escolaridade inferior a } 8 \text { anos }(\mathrm{OR}=2,4 ; \mathrm{IC}=1,3-4,3) \text {, histórico } \\
\text { prévio de abandono de tratamento }(\mathrm{OR}=3,3 ; \mathrm{IC}=1,6-7,7) .\end{array}$ \\
\hline $\begin{array}{l}\text { Hoa et al. } \\
\text { (17) }\end{array}$ & Vietnã & 2011 & $\begin{array}{l}\text { Inquérito } \\
\text { (populacional) }\end{array}$ & 87413 & Prevalência & $\begin{array}{l}\text { Despesas familiares com bens de consumo: Quintil mais baixo } \\
\quad(R R=2,5 ; I C=1,6-3,9) \text {. }\end{array}$ \\
\hline
\end{tabular}

a $\mathrm{AOR}$ = razão de chances ajustada; IC = intervalo de confiança; $\mathrm{OR}$ = razão de chances; $\mathrm{RR}$ = risco relativo.

para as variáveis analfabetismo, alcoolismo, faixa etária de 30 a 64 anos, ter recebido tratamento prévio, sintomas clínicos desfavoráveis após 4 semanas do início do tratamento, histórico prisional e ser fumante $(14,15)$. Quanto à falha terapêutica, apresentaram associação direta as variáveis referentes ao atraso no início do tratamento, analfabetismo e alcoolismo (14). A análise multivariada para o conjunto de desfechos negativos (óbito/ abandono/falha terapêutica) apontou para associação direta com escolaridade (até 8 anos de estudo), histórico prévio de abandono de tratamento, coinfecção com HIV e alcoolismo $(14,16)$.

Nos inquéritos, a prevalência de casos foi associada com baixo nível de despesas familiares com bens do tipo televisão, rádio, automóvel, tipo de combustível utilizado para cozinhar, renda inferior a 1 dólar por dia, não possuir título de posse da terra e viver em habitações com precariedade estrutural $(17,18)$. Quando a variável dependente foi atraso por parte do paciente na busca por diagnóstico de tuberculose, verificou-se associação direta com idade entre 40 e 59 anos e baixo nível educacional (19). Para a mortalidade foi verificada associação significativa com a variável escolaridade inferior a 3 anos de estudo, abandono de tratamento e não ter recebido tratamento diretamente observado (DOT) (20).

\section{Estudos ecológicos}

Do total de 25 estudos de nível ecológico selecionados para revisão, um trabalho buscou associação tendo regiões continentais (conjunto de países) como nível de agregação espacial dos dados e outro utilizou dados agregados por país. Além disso, dois estudos enfocaram províncias ou estados, um enfocou municípios, nove enfocaram distritos administrativos, seis enfocaram bairros e cinco enfocaram setores censitários (tabela 2).

A análise da associação entre a variação anual da taxa de incidência de tuberculose e indicadores demográficos, econômicos e de serviço de saúde para o conjunto de 134 países distribuídos ao longo de cinco continentes aponta para uma relação inversa entre incidência de tuberculose e aumento do produto interno bruto (PIB), índice de desenvolvimento humano (IDH), acesso a saneamento básico e baixa mortalidade infantil $(4,21)$. 
TABELA 2. Estudos ecológicos sobre associação entre fatores socioeconômicos e tuberculose

\begin{tabular}{|c|c|c|c|c|c|}
\hline Referência & País & Ano & $\begin{array}{l}\text { Agregação } \\
\text { dos dados }\end{array}$ & $\begin{array}{c}\text { Variável } \\
\text { dependente }\end{array}$ & Indicadores associados ${ }^{a}$ \\
\hline $\begin{array}{l}\text { Spence et al. } \\
\text { (25) }\end{array}$ & Inglaterra & 1993 & $\begin{array}{l}\text { Distritos } \\
\text { administrativos }\end{array}$ & Incidência & Índices de Jarman $(r=0,73 ; \mathrm{IC}=0,5-0,8)$ e Índice de Townsend $(r=0,6 ; \mathrm{IC}=0,3-0,7)$. \\
\hline $\begin{array}{l}\text { Mangtani et al. } \\
\text { (34) }\end{array}$ & Inglaterra & 1995 & Bairros & Incidência & $\begin{array}{l}\text { Densidade intradomiciliar (aumento de } 1 \% \text { resultou em variação de } 12 \% \text { na taxa média de } \\
\text { incidência; IC = 4,8-19,4), proporção de imigrantes (aumento de } 1 \% \text { resultou em variação } \\
\text { de } 5 \% \text { na taxa média de incidência; IC }=2,7-8,1 \text { ). }\end{array}$ \\
\hline $\begin{array}{l}\text { Cantwell et al. } \\
\qquad(23)\end{array}$ & EUA & 1997 & Estados & Incidência & $\begin{array}{l}\text { Densidade intradomiciliar (razão de taxa entre } 4^{\circ} \text { e } 1^{\circ} \text { quartil socioeconômico }=1,7 \text { ), pessoas } \\
\text { vivendo abaixo da linha de pobreza (razão de taxa }=1,5) \text {, percentual de pessoas recebendo } \\
\text { assistência governamental relativa à renda (razão de taxa }=1,2 \text { ) e percentual de pessoas } \\
\text { desempregadas (razão de taxa }=1,2 \text { ). Todos indicadores apresentaram } P<0,01 \text {. }\end{array}$ \\
\hline $\begin{array}{l}\text { Hawker et al. } \\
\text { (24) }\end{array}$ & Inglaterra & 1999 & $\begin{array}{l}\text { Distritos } \\
\text { administrativos }\end{array}$ & Incidência & $\begin{array}{l}\text { Proporção de desempregados, domicílios com mais de 1,5 moradores por cômodo } \\
\text { (magnitude da associação não apresentada; } P<0,01 \text { ). }\end{array}$ \\
\hline $\begin{array}{l}\text { Tocque et al. } \\
\text { (29) }\end{array}$ & Inglaterra & 1999 & $\begin{array}{l}\text { Distritos } \\
\text { administrativos }\end{array}$ & Incidência & $\begin{array}{l}\text { Densidade populacional }\left(r^{2}=0,37 ; P<0,001\right) \text {, desemprego }\left(r^{2}=0,58 ; P<0,001\right) \text {, proporção } \\
\text { de idosos morando sozinhos }(r=0,51 ; P<0,001) \text {. }\end{array}$ \\
\hline $\begin{array}{l}\text { Bennett et al. } \\
\qquad(30)\end{array}$ & Inglaterra & 2001 & $\begin{array}{l}\text { Distritos } \\
\text { administrativos }\end{array}$ & Incidência & $\begin{array}{l}\text { Densidade intradomiciliar }\left(r^{2}=0,23 ; I C=12,0-36,0\right) \text {, imigrantes }\left(r^{2}=0,5 ; I C=41,0-58,0\right) \text { e } \\
\text { proporção de pessoas sem a posse da habitação }\left(r^{2}=0,27 ; I C=18,0-35,0\right)\end{array}$ \\
\hline $\begin{array}{l}\text { Barr et al. } \\
\text { (33) }\end{array}$ & EUA & 2001 & Bairros & Incidência & $\begin{array}{l}\text { Densidade intradomiciliar ( } R R=1,3 ; I C=1,2-1,4) \text {, proporção de pessoas com baixo } \\
\text { rendimento monetário ( } R R=1,3 ; I C=1,3-1,4) \text {, incidência de AIDS ( } R R=1,4 ; I C=1,4-1,5) \text {. }\end{array}$ \\
\hline $\begin{array}{l}\text { Quijano et al. } \\
\text { (35) }\end{array}$ & Espanha & 2001 & Bairros & Incidência & $\begin{array}{l}\left.\text { Desemprego (Bivariada } \mathrm{r}^{2}=0,8 P<0,05 \text {; Multivariada } \mathrm{RR}=1,18 ; \mathrm{IC}=1,1-1,3\right) \text {, baixa } \\
\text { escolaridade }\left(\mathrm{r}^{2}=0,74 ; P<0,05\right) \text {, densidade intradomiciliar }\left(\mathrm{r}^{2}=0,52 ; P<0,05\right) \text {, habitar } \\
\text { bairros da zona central com piores condições de vida }(\mathrm{RR}=1,8 ; \mathrm{IC}=1,5-2,1)\end{array}$ \\
\hline $\begin{array}{l}\text { Vincentin et al. } \\
\qquad(10)\end{array}$ & Brasil & 2002 & $\begin{array}{l}\text { Distritos } \\
\text { administrativos }\end{array}$ & Mortalidade & $\begin{array}{l}\text { Índice de Robin Hood }(r=0,44) \text {, razão da renda entre os } 10 \% \text { mais ricos e } 40 \% \text { mais pobres } \\
(r=0,42) \text { e chefes de domicílio com renda entre um e dois salários mínimos }(r=0,5) \text {. } \\
\text { Escolaridade de nível superior }(r=-0,48) \text {, área média por domicílio }(r=-0,5) \text { e número } \\
\text { médio de cômodos por domicílio }(r=-0,72) \text {, Todos indicadores apresentaram } P<0,05 \text {. }\end{array}$ \\
\hline $\begin{array}{l}\text { Nishiura et al. } \\
\qquad(26)\end{array}$ & Japão & 2003 & $\begin{array}{l}\text { Distritos } \\
\text { administrativos }\end{array}$ & Incidência & $\begin{array}{l}\text { Densidade populacional, proporção de domicílios com ajuda governamental, domicílio com área } \\
\text { habitacional menor que a padrão (magnitude da associação não apresentada; } P<0,01 \text { ). }\end{array}$ \\
\hline $\begin{array}{l}\text { Serpa et al. } \\
\quad(32)\end{array}$ & Cuba & 2003 & Bairros & Incidência & $\begin{array}{l}\text { Densidade intradomiciliar, percentual de famílias com carência econômica e de saneamento } \\
\text { básico (magnitude da associação não apresentada; } P<0,01 \text { ). }\end{array}$ \\
\hline $\begin{array}{l}\text { Leung et al. } \\
\qquad(27)\end{array}$ & China & 2004 & $\begin{array}{l}\text { Distritos } \\
\text { administrativos }\end{array}$ & Incidência & $\begin{array}{l}\text { Percentual de viúvos ou divorciados }(r=0,50 ; P=0,02) \text {, pessoas por cômodo }(r=0,67 \\
\qquad P=0,002) \text {. }\end{array}$ \\
\hline Liu JJ (22) & China & 2005 & Províncias & Prevalência & $\begin{array}{l}\text { Renda liquida per capita da população rural }(r=-0,42 ; P<0,05) \text {, PIB per capita }(r=-0,47 \text {; } \\
\qquad P<0,05) \text { e número de médicos por } 1000 \text { habitantes }(r=-0,47 ; P<0,05) \text {. }\end{array}$ \\
\hline $\begin{array}{l}\text { Chan-Yeung } \\
\text { et al. (38) }\end{array}$ & China & 2005 & $\begin{array}{l}\text { Setores } \\
\text { censitários }\end{array}$ & Incidência & $\begin{array}{l}\text { Proporção de população com mais de } 65 \text { anos }(r=0,45 ; P<0,05) \text {, pessoas com baixa renda } \\
\qquad(r=0,74 ; P<0,05) \text {, pessoas acima de } 15 \text { anos com baixa escolaridade }(r=0,53 ; P<0,05) \text {. }\end{array}$ \\
\hline $\begin{array}{l}\text { Ponticiello et al. } \\
\qquad(36)\end{array}$ & Itália & 2005 & Bairros & Incidência & $\begin{array}{l}\text { Densidade populacional, pessoas analfabetas, desemprego (magnitude da associação não } \\
\text { apresentada; } P<0,05) \text {. }\end{array}$ \\
\hline $\begin{array}{l}\text { Myers et al. } \\
\text { (39) }\end{array}$ & EUA & 2006 & $\begin{array}{l}\text { Setores } \\
\text { censitários }\end{array}$ & Incidência & $\begin{array}{l}\text { Proporção de imigrantes (razão de taxa }=1,6 ; I C=1,6-1,7 \text { ), domicílios com mais de um } \\
\text { morador por cômodo (razão de taxa }=1,6 ; I C=1,5-1,6 \text { ), pessoas maiores de } 25 \text { anos } \\
\text { sem o segundo grau escolar (razão de taxa }=1,7 ; I C=1,6-1,7 \text { ), maiores de } 16 \text { anos sem } \\
\text { emprego (razão de taxa }=1,4 ; I C=1,4-1,5) .\end{array}$ \\
\hline $\begin{array}{l}\text { Souza et al. } \\
\qquad(40)\end{array}$ & Brasil & 2007 & $\begin{array}{l}\text { Setores } \\
\text { censitários }\end{array}$ & Incidência & $\begin{array}{l}\text { Moradores por domicílio }(R R=1,94 ; I C=1,1-3,5) \text {, casos de retratamento por setor censitário } \\
(R R=1,8 ; I C=1,4-2,2) \text { e famílias com mais de um caso por setor censitário }(R R=1,9 ; \\
I C=1,6-2,2) \text {. }\end{array}$ \\
\hline $\begin{array}{l}\text { Silva et al. } \\
\quad(37)\end{array}$ & Brasil & 2008 & Bairros & Mortalidade & $\begin{array}{l}\text { Índice composto de condições socioeconômicas: acesso precário a saneamento básico, } \\
\text { chefes de domicílios com até } 3 \text { anos de estudo e chefes de domicílio com renda até dois } \\
\text { salários mínimos }(r=0,32 ; P<0,01) \text {. }\end{array}$ \\
\hline $\begin{array}{l}\text { Janssens et al. } \\
\qquad(21)\end{array}$ & Suíça & 2008 & País & Incidência & $\begin{array}{l}\text { PIB per capita (aumento de duas vezes no PIB associa-se a uma diminuição de 38,5\% na } \\
\text { incidência de tuberculose) (estatísticas de associação não apresentada). }\end{array}$ \\
\hline Fasca et al. (3) & Brasil & 2008 & Municípios & Incidência & $\begin{array}{l}\text { Densidade de pobres }(r=0,61 ; P=0,000) \text {, incidência de AIDS }(r=0,54 ; P=0,000) \text {, Gini da } \\
\quad \text { renda }(r=-0,32 ; P=0,001) \text {. }\end{array}$ \\
\hline Angelo et al. (31) & Brasil & 2008 & $\begin{array}{l}\text { Distritos } \\
\text { administrativos }\end{array}$ & Incidência & $\begin{array}{l}\text { Densidade de pobres }(r=0,5 ; P<0,05) \text {, incidência de AIDS }(r=0,6 ; P<0,05) \text {, valor médio } \\
\text { do imposto territorial urbano }(r=0,5 ; P<0,05) \text {. }\end{array}$ \\
\hline Dye et al. (4) & Suíça & 2009 & $\begin{array}{l}\text { Regiões } \\
\text { continentais } \\
\text { (conjunto de } \\
\text { países) }\end{array}$ & Incidência & $\begin{array}{l}\text { Prevalência de HIV entre pacientes de tuberculose }(r=0,26) \text {, deficiência alimentar }(r=0,36) \text {, } \\
\text { mortalidade em }<\text { de } 5 \text { anos }(r=0,36) \text {, baixo rendimento econômico }(r=0,43) \text {, aumento } \\
\text { do PIB }(r=-0,21) \text {, aumento do IDH }(r=-0,60) \text {, acesso a saneamento básico }(r=-0,43) \text {, } \\
\text { percentual do PIB gasto em despesas totais em saúde }(r=-0,53) \text {. Todos indicadores } \\
\text { apresentaram } P<0,01 \text {. }\end{array}$ \\
\hline $\begin{array}{l}\text { Ximenes et al. } \\
(6)\end{array}$ & Brasil & 2009 & $\begin{array}{l}\text { Setores } \\
\text { censitários }\end{array}$ & Incidência & $\begin{array}{l}\text { Domicílios com mais de quatro moradores }(\mathrm{OR}=1,83 ; \mathrm{IC}=1,3-2,5) \text {, percentual de pessoas } \\
\text { alfabetizadas }(\mathrm{OR}=0,66 ; \mathrm{IC}=0,4-0,8) \text {, percentual de pessoas empregadas }(\mathrm{OR}=0,47 \\
\mathrm{IC}=0,3-0,7) \text {, percentual de casas com refrigerador, máquina de lavar, computador } \mathrm{e} \\
\text { ar-condicionado }(\mathrm{OR}=0,52 ; \mathrm{IC}=0,4-0,7) \text {. }\end{array}$ \\
\hline $\begin{array}{l}\text { Pang et al. } \\
\text { (28) }\end{array}$ & China & 2010 & $\begin{array}{l}\text { Distritos } \\
\text { administrativos }\end{array}$ & Incidência & $\begin{array}{l}\text { Proporção de indivíduos não casados }(r=0,17 ; P<0,01) \text {, escolaridade até } 4 \text { anos }(r=0,45 \text {; } \\
\quad P<0,01) \text {, pessoas com baixo rendimento monetário }(r=0,26 ; P<0,01) \text {. }\end{array}$ \\
\hline $\begin{array}{l}\text { Vendramini et al. } \\
\text { (41) }\end{array}$ & Brasil & 2010 & $\begin{array}{l}\text { Setores } \\
\text { censitários }\end{array}$ & $\begin{array}{c}\text { Coinfecção } \\
\text { TB/HIV }\end{array}$ & $\begin{array}{l}\text { Chefes de domicílio com até } 3 \text { anos de estudo, proporção de mulheres analfabetas, } \\
\text { proporção de pessoas com renda entre dois e três salários mínimos (magnitude da } \\
\text { associação não apresentada; } P<0,05 \text { ). }\end{array}$ \\
\hline
\end{tabular}

a IC = intervalo de confiança; IDH = índice de desenvolvimento humano; PIB = produto interno bruto. 
Quando consideradas as regiões de análise propostas pela Organização Mundial da Saúde (OMS), observa-se, para a região da América Latina e Caribe, associação direta entre a variação ao longo dos anos da taxa de incidência de tuberculose e a prevalência de HIV, prevalência de HIV entre pacientes de tuberculose, pessoas com deficiência alimentar e variação anual da mortalidade em crianças menores de 5 anos. Associação inversa foi verificada para PIB per capita e para pessoas com acesso a serviços de abastecimento de água e esgotamento sanitário (4).

Para a região mediterrânea oriental (Afeganistão, Arábia Saudita, Egito, Emirados Árabes, Irã, Iraque, Jordânia, Líbano, Líbia, Marrocos, Paquistão, Síria, Tunísia), a variação anual da taxa de incidência associa-se inversamente com o percentual do PIB gasto em despesas totais em saúde. Quanto aos países de alta renda (Alemanha, Austrália, Canadá, Espanha, Estados Unidos, França, Inglaterra, Itália, Japão, Portugal) a incidência de tuberculose reduziu-se mais rapidamente entre aqueles com menor proporção de pacientes de origem estrangeira. Em relação aos fatores associados diretamente na região da Europa Central e Oriental, destacam-se a variação anual da mortalidade em crianças menores de 5 anos, pessoas com baixo rendimento econômico e deficiência alimentar. Ainda para essa região, observa-se associação inversa com IDH, PIB per capita, gastos totais em saúde per capita e percentual do PIB gasto em despesas totais em saúde (4).

Estudo de associação entre prevalência de tuberculose e indicadores socioeconômicos, demográficos e de serviços de saúde utilizando províncias como nível de agregação espacial dos dados apontou para relação inversa referente à renda liquida per capita da população rural, PIB per capita e número de médicos por 1000 habitantes (22).

Quanto ao nível de agregação espacial dos dados referente a estados, um estudo verificou associação direta entre incidência de tuberculose e as seguintes variáveis socioeconômicas: número médio de pessoas por cômodo, percentual de pessoas vivendo abaixo da linha de pobreza, percentual de pessoas recebendo assistência governamental relativa à renda e percentual de pessoas desempregadas. Para os autores, o risco relativo à tuberculose aumenta consi- deravelmente nos estratos de baixa situação socioeconômica comparando ao quartil mais elevado (23).

Em relação à utilização de municípios como nível de agregação espacial dos dados, um estudo verificou associação direta entre a incidência de tuberculose e a densidade de pobres (moradores com rendimento mensal inferior a 2 salários mínimos brasileiros dividido pela área total utilizável em $\mathrm{km}^{2}$ ), assim como incidência de AIDS. Associação inversa foi observada para o Índice de Gini relativo à renda (3).

Os estudos que buscaram analisar a variação na taxa de incidência de tuberculose e fatores socioeconômicos tendo como nível de agregação espacial dos dados os distritos administrativos observaram associação direta com as seguintes variáveis: densidade de pobres, incidência de AIDS, proporção de residentes desempregados, domicílios com mais de 1,5 morador por cômodo, índices desfavoráveis de Jarman e Townsend (pobreza e privação), densidade populacional, proporção de indivíduos não casados, pessoas com escolaridade até 4 anos, pessoas com baixo rendimento monetário, percentual de viúvos ou divorciados, valor médio do imposto territorial urbano, imigração, proporção de pessoas sem a posse da habitação (24-31).

Em relação à mortalidade por tuberculose, um estudo verificou associação direta com índice de Robin Hood (proporção de renda que deveria ser retirada dos ricos e transferida para os pobres de forma a se obter uma distribuição equitativa), razão da renda média entre os $10 \%$ mais ricos e os $40 \%$ mais pobres e proporção de chefes de domicílio com renda média entre um e dois salários mínimos. Associação inversa foi observada para as variáveis relativas a escolaridade de nível superior, área média por domicílio e número médio de cômodos por domicílio (10).

Quanto aos estudos que buscaram correlação entre modificações nas taxas de incidência de tuberculose e indicadores socioeconômicos ao nível de bairros, foi observada associação direta com variáveis referentes a densidade intradomiciliar, percentual de famílias com carência econômica e de saneamento básico, pessoas com baixo rendimento monetário, incidência de AIDS, desemprego, imigração, proporção de pessoas com baixa escolaridade (32-36). Em relação à mortalidade por tuberculose, um estudo verificou associação direta com o acesso precário a saneamento básico, proporção de pessoas analfabetas, chefes de domicílios com até 3 anos de estudo e chefes de domicílio com renda até dois salários mínimos (37).

Os estudos que buscaram investigar a relação entre fatores socioeconômicos e a incidência de tuberculose utilizando os setores censitários como nível de agregação espacial dos dados apontaram para associação direta com as seguintes variáveis: domicílios com mais de cinco moradores, analfabetismo, proporção de população com mais de 65 anos, proporção de pessoas com baixa renda, proporção de pessoas acima de 15 anos com baixa escolaridade, proporção de imigrantes, domicílios com mais de um morador por cômodo, números de casos de retratamento por setor censitário e número de famílias com mais de um caso por setor censitário $(6,38-40)$.

Um estudo utilizando como variável dependente a coinfecção tuberculose/ HIV destacou a associação positiva com indicadores socioeconômicos referentes a chefes de família com até 3 anos de estudo, proporção de mulheres analfabetas e proporção de pessoas com renda entre dois e três salários mínimos (41).

\section{DISCUSSÃO}

A análise dos estudos selecionados na presente revisão bibliográfica aponta para a persistência da relação entre fatores socioeconômicos e o processo de produção da tuberculose tanto em nível individuado quanto ao nível coletivo.

Um dos poucos estudos utilizando modelo multinível demonstrou que, em relação à tuberculose, não há interação entre fatores socioeconômicos em nível individuado e ecológico. A independência entre os dois níveis de análise sugere que o risco individuado de desenvolver tuberculose aumenta de acordo com as características socioeconômicas da população de seu entorno (6).

Em relação aos estudos de nível individuado, foi possível observar que fatores como alcoolismo, baixo nível escolar, baixo rendimento monetário, carência alimentar e coinfecção pelo vírus HIV associam-se a múltiplos desfechos relacionados ao acometimento por tuberculose (tabela 1).

Os indicadores socioeconômicos referentes aos baixos níveis de renda e escolaridade poderiam aumentar a vulnera- 
bilidade à tuberculose ao refletir o acesso individual e desigual à informação, a benefícios oriundos do conhecimento, aos bens de consumo e ao serviço de saúde. Quanto à carência alimentar, dietas com baixo valor proteico relacionam-se a alterações na função imunológica mediada por células $\mathrm{T}$, tornando o organismo mais suscetível à infecção por Mycobacterium tuberculosis e ao desenvolvimento da doença $(13,25)$.

Variáveis relativas ao sexo (masculino), faixa etária (entre 25 e 49 anos) e estado civil (solteiro, viúvo ou divorciado) também se associaram de forma significativa à tuberculose em nível individuado $(12,19)$. O maior acometimento de indivíduos com tais características poderia ser reflexo da mobilidade espacial, resultando em maiores taxas de contato social. Pang et al. (28) sugerem que o estado civil (solteiro, viúvo ou divorciado) poderia implicar na ausência ou no menor grau de apoio familiar, aumentando a vulnerabilidade à tuberculose em momentos de estresse psicossocial.

Em nível ecológico, foi possível verificar que indicadores referentes a renda, escolaridade e densidade populacional associam-se a tuberculose nos distintos níveis de agregação espacial (tabela 2). Barr et al. (33) observaram que o acréscimo de $10 \%$ na proporção de famílias vivendo com renda abaixo da linha de pobreza esteve associado com um aumento de $33 \%$ na taxa de incidência de tuberculose para os bairros de Nova Iorque no período de 1984 a 1992. Magnati et al. (34) verificaram que o aumento de $1 \%$ na proporção de domicílios com mais de uma pessoa por cômodo representou um aumento de $12 \%$ na taxa média de notificação de tuberculose para os bairros de Londres entre 1982 e 1991.

Apesar da relação existente entre tuberculose e indicadores socioeconômicos, a associação entre esses parece ser influenciada tanto pelo nível de agregação espacial dos dados quanto pelas características particulares das áreas geográficas em estudo. Vicentim et al. (10) destacam que a fraca associação entre densidade demográfica e mortalidade por tuberculose nas regiões administrativas (RA) do Município do Rio de Janeiro pode resultar do fato de que grandes densidades são encontradas tanto em RAs com elevada mortalidade por tuberculose quanto nas de baixo coeficiente, não permitindo, desse modo, uma distinção mais direta. Pensamento análogo pode ser assumido em relação a indicadores como proporção de pobres e analfabetismo $(3,10,32)$.

Ainda quanto à influência das características particulares das áreas geográficas, Fasca et al. (3) chamam a atenção para a utilização de indicadores socioeconômicos capazes de agregar atributos individuais e espaciais, a exemplo da densidade de pobres. Uma vez que a área geográfica total é substituída pela área utilizável, na qual efetivamente as pessoas habitam, trabalham ou transitam, esse tipo de indicador mostrou-se mais sensível do que a proporção de pobres, assim como a densidade demográfica na definição de espaços de maior vulnerabilidade social à tuberculose. Para tais autores, o indicador densidade de pobres (assim como outros indicadores socioeconômicos sintéticos) não é suficiente para explicar sozinho a transmissão da doença, sugerindo a existência de particularidades inerentes a cada unidade territorial analisada. Nesse sentido, destaca-se que a pobreza é um fenômeno heterogêneo, com grande variação de tipo e magnitude entre países, regiões ou mesmo bairros. Indicadores socioeconômicos e epidemiológicos não atuam de forma isolada, mas segundo características conjunturais próprias que devem ser consideradas nas análises (42).

Em relação às limitações desta revisão bibliográfica, a estratégia de inclusão adotada não foi capaz de captar os trabalhos em cujo resumo não havia nenhuma referência sobre possível relação estatística de associação. É possível que a maior representatividade dos estudos realizados no Brasil se deva à não inclusão de trabalhos de acesso restrito como artigos, teses e dissertações realizadas em outros países, assim como à não inclusão de estudos publicados em idiomas oficiais de países como China e Índia.

Quanto ao conjunto de estudos ecológicos, os artigos selecionados para revisão sistemática apresentaram uma clara descrição metodológica, com explicitação da população e unidades geográficas, fonte de dados e construção das variáveis independentes e modelo utilizado na análise. A maioria (68\%) fez uso de regressão multivariada para estimação de associação, controle de con- fundimento e interações das variáveis independentes. Porém, poucos estudos (12\%) levaram em consideração a existência de uma possível dependência espacial entre as unidades geográficas, assim como procedimentos para correção das taxas estimadas em decorrência da provável flutuação referente a unidades geográficas com pequenas populações (distritos, bairros e setores censitários).

Os estudos observacionais incluídos nesta revisão sistemática apresentaram uma clara definição dos critérios de elegibilidade para casos e controles, tamanho amostral, explicitação de perdas de segmento, descrição das variáveis independentes, modelos de análise, medidas de associação brutas e ajustadas e respectivos intervalos de confiança ou P-valor.

As principais limitações destacadas nos estudos tipo caso-controle referemse a possível viés de informação (qualidade de informações de fonte secundária e ao processo de coleta primária de dados) e seleção e controle de variáveis de confundimento. Nos estudos de coorte, a principal limitação destacada foi referente ao controle de possíveis variáveis de confundimento. Nos trabalhos com delineamento seccional foram mencionados possíveis vieses de informação decorrentes do instrumento de coleta e a qualidade da informação de dados secundários.

Por fim, ao analisar os estudos de associação entre tuberculose e fatores socioeconômicos oriundos de diferentes países, destacamos que a tuberculose continua a ser uma doença cujo processo de produção encontra-se intimamente relacionado às condições de vida. Além do diagnóstico precoce, tratamento adequado e vigilância epidemiológica, a diminuição das desigualdades socioeconômicas e a adequação dos programas de controle às realidades locais em que atuam figuram como fatores primordiais na redução da mortalidade e morbidade por tuberculose.

Agradecimentos. ASP recebeu bolsa de doutorado da Coordenação de Aperfeiçoamento de Pessoal de Nível Superior (CAPES) durante o período do estudo.

Conflito de interesses. Nada declarado pelos autores. 


\section{REFERÊNCIAS}

1. World Health Organization (WHO). Global tuberculosis control report. Genebra: WHO; 2011. (WHO/HTM/TB/2011.16)

2. Lonnroth K, Castro KG, Chakaya JM, Chauhan LS, Floyd K, Glaziou P, et al. Tuberculosis control and elimination 2010-50: cure, care and social development. Lancet. 2010;375(9728):1814-29.

3. Fasca SF. Tuberculose e condições de vida: uma análise do Estado do Rio de Janeiro, Brasil, 2000 a 2002 [dissertação]. Rio de Janeiro: Escola Nacional de Saúde Pública, Fundação Oswaldo Cruz; 2008.

4. Dye C, Lonnroth K, Jaramillo E, William BG, Raviglione M. Trends in tuberculosis incidence and their determinants in 134 countries. Bull Word Health Organ. 2009;87(9):683-91.

5. Oxlade O, Schwartzman K, Behr MA, Beneditti A, Pai M, Heymann J, et al. Global tuberculosis trends: a reflection of changes in tuberculosis control or in population health? Int J Tuberc Lung Dis. 2009;13(10):1238-46.

6. de Alencar Ximenes RA, de Fátima Pessoa Militão de Albuquerque M, Souza WV, Montarroyos UR, Diniz GT, Luna CF, et al. Is it better to be rich in a poor area or poor in a rich area? A multilevel analysis of a case-control study of social determinants of tuberculosis. Int J Epidemiol. 2009;38(5):1285-96.

7. Pelaquin MHH, Silva RS, Ribeiro AS. Fatores associados ao óbito por tuberculose na zona leste da cidade de São Paulo, 2001. J Bras Pneumol. 2007;33(3):311-7.

8. Sánchez-Pérez H, Flores-Hernández J, Jansá J, Caylá J, Martín-Mateo M. Pulmonary tuberculosis and associated factors in areas with high levels of poverty in Chiapas, Mexico. Int J Epidemiol. 2001;30(2):386-93.

9. Spence DP, Hotchkiss J, Williams CS, Davies PD. Tuberculosis and poverty. BMJ. 1993;307(6907):759-61.

10. Vincentin G, Santo AH, Carvalho MS. Mortalidade por tuberculose e indicadores sociais no município do Rio de Janeiro. Cienc Saude Coletiva. 2002;7(2):253-63.

11. Vandenbroucke JP, von Elm E, Altman DG, Gotzsche PC, Mulrow CD, Pocock SJ, et al. Strengthening the Reporting of Observational Studies in Epidemiology (STROBE): explanation and elaboration. PLoS Med. 2011;4(10):e297.

12. Tekkel M, Rahu M, Loit HM, Baburim A. Risk factors for pulmonary tuberculosis in Estonia. Int J Tuberc Lung Dis. 2002;6(10):887-94.

13. Bocia D, Hargreaves J, De Stavola BL, Fielding K, Schaap A, Godfrey-Faussett P, et al. The association between household socioeconomic position and prevalent tuberculosis in Zambia: a case-control study. PLoS One. 2011;6(6):e20824.

14. de Albuquerque MF, Ximenes RA, LucenaSilva N, de Sousa WV, Dantas AT, Dantas $\mathrm{OM}$, et al. Factors associated with treatment failure, dropout, and death in a cohort of tuberculosis patients in Recife, Pernambuco State, Brazil. Cad Saude Publica. 2007;23(7):1573-82.

15. Kittikraisak W, Burapat C, Kaewsa-ard S, Watthanaamornkiet W, Sirinak C, Sattayawuthipong $\mathrm{W}$, et al. Factors associated with tuberculosis treatment default among HIV-infection tuberculosis patients in Thailand. Trans R Soc Trop Med Hyg. 2009;103(1): 59-66.

16. Belo MT, Luiz RR, Teixeira EG, Hanson C, Trajman A. Tuberculosis treatment outcomes and socioeconomic status: a prospective study in Duque de Caxias, Brazil. Int J Tuberc Lung Dis. 2011;15(7):978-81.

17. Hoa NB, Tiemersma EW, Sy DN, Nhung NV, Gebhad A, Borgdorff MW, et al. Household expenditure and tuberculosis prevalence in VietNam: prediction by a set of household indicators. Int J Tuberc Lung Dis. 2011;15(1): 32-7.

18. Muniyandi M, Ramachandran R, Gopi PG, Chandrasekaran V, Subramani R, Sadacharam K, et al. The prevalence of tuberculosis in different socioeconomic strata: a community survey from South India. Int J Tuberc Lung Dis. 2007;11(9):1042-5.

19. Cheng G, Tolhurst R, Li RZ, Meng QY, Tang $S$. Factors affecting delays in tuberculosis diagnosis in rural China: a case study in four counties in Shandong Province. Trans R Soc Trop Med Hyg. 2005;99(5):355-62.

20. Nájera-Ortiz JC, Sánches-Pérez HJ, OchoaDíaz $\mathrm{H}$, Arana-Cedeño $\mathrm{M}$, Lezama MS, Mateo MM. Demographic, health service and socioeconomic factors associated with pulmonary tuberculosis mortality in Los Altos Region of Chipas, Mexico. Int J Epidemiol. 2008;37(4):786-95

21. Janssens JP, Rieder HL. An ecologic analysis of incidence of tuberculosis and per capita gross domestic product. Eur Respir J. 2008;32(5):1415-6.

22. Liu JJ, Yao HY, Liu EY. Analysis of factors affecting the epidemiology of tuberculosis in China. Int J Tuberc Lung Dis. 2005;9(4):450-4.

23. Cantwell MF, McKenna MT, McRay E, Onorato IM. Tuberculosis and race/ethnicity in the United States: impact of socioeconomic status. Am J Respir Crit Care Med. 1997;157 (4 Pt 1):1016-20.

24. Hawker JI, Bakhshi SS, Ali S, Farrington CP. Ecological analysis of ethnic differences in relation between tuberculosis and poverty. BMJ. 1999;319(7216):1031-4.

25. Spence DP, Hotchkiss J, William CS, Davies PD. Tuberculosis and poverty. BMJ. 1993;307(6907):759-61.

26. Nishiura H. [Socioeconomic factors for tuberculosis in Tokyo, Japan-unemployment, overcrowding, poverty and migrants] Kekkaku. 2003;78(6):419-26.

27. Leung CC, Yew WW, Tam CM, Chang KC, Chan CK, Law WS, et al. Socioeconomic factors and tuberculosis: a district-base ecological analysis in Hong Kong. Int J Tuberc Lung Dis. 2004;8(8):958-64.

28. Pang PT, Leung CC, Lee SS. Neighbourhood risk factors for tuberculosis in Hong Kong. Int J Tuberc Lung Dis. 2010;14(5):585-92.

29. Tocque $K$, Regan M, Remmington T, Beeching NJ, Jamieson I, Syed Q, et al. Social factors associated with increases in tuberculosis notification. Eur Respir J. 1999;13(3):541-5.

30. Bennett J, Pitman R, Jarman B, Innes J, Best N, Alves B, et al. A study of the variation in tu- berculosis incidence and possible influential variables in Manchester, Liverpool, Birmingham and Cardiff in 1991-1995. Int J Tuberc Lung Dis. 2001;5(2):158-63.

31. Angelo JR. (Re)Produção do espaço urbano de Juiz de Fora e a distribuição espacial da tuberculose [dissertação]. Rio de Janeiro: Escola Nacional de Saúde Pública, Fundação Oswaldo Cruz; 2008

32. Serpa IM, Pardo CL, Hernandez RA. Un estudio ecológico sobre tuberculosis en un municipio de Cuba. Cad Saude Publica. 2003;19(5):1305-12.

33. Barr RG, Diez-Roux AV, Knirsch CA, Pablos-Mendez A. Neighborhood poverty and the resurgence of tuberculosis in New York City, 1984-1992. Am J Public Health. 2001; 91(9):1487-93.

34. Mangtani P, Jolley DJ, Watson JM, Rodrigues LC. Socioeconomic deprivation and notification rates for tuberculosis in London during 1982-1991. BMJ. 1995;310(6985):963-6.

35. Diaz de Quijano E, Brugal MT, Pasarin MI, Galdos-Tanguis H, Cayla J, Borrrel C. Influencia de las desigualdades sociales, la conflictividad social y la pobreza extrema sobre la morbidad por tuberculosis en la ciudad de Barcelona. Rev Esp Salud Publica. 2001;75(6):517-27.

36. Ponticiello A, Sturkenboom MC, Simonetti A, Ortolani R, Malerba M, Sanduzzi A. Deprivation, immigration and tuberculosis incidence in Naples, 1996-2000. Eur J Epidemiol. 2005;20(8):729-34.

37. Silva VL, Leal MCC, Marino JG, Marques APO. Associação entre carência social e causas de morte entre idosos residentes no município de Recife, Pernambuco, Brasil. Cad Saude Publica. 2008;24(5):1013-23.

38. Chan-yeung M, Yeh AG, Tam CM, Kam $\mathrm{KM}$, Leung CC, Yew WW, et al. Sociodemographic and geographic indicators and distribution of tuberculosis in Hong Kong: a spatial analysis. Int $\mathrm{J}$ Tuberc Lung Dis. 2005;9(12):1320-6.

39. Myers WP, Westenhouse JL, Flood J, Riley LW. An ecological study of tuberculosis transmission in California. Am J Public Health. 2006;96(4):685-90.

40. Souza WV, Carvalho MS, Albuquerque MF, Barcellos CC, Ximenes RA. Tuberculosis in intra-urban settings: a Bayesian approach Trop Med Int Health. 2007;12(3):323-30.

41. Vendramini SHF, Santos NSGM, Santos MLSG, Chiaravalloti-Neto F, Ponce MAZ, Gazetta $\mathrm{CE}$, et al. Análise espacial da coinfecção tuberculose/HIV: relação com níveis socioeconômicos em município do sudeste do Brasil. Rev Soc Bras Med Trop. 2010; 43(5):536-41.

42. Barcellos C. Os indicadores da pobreza e a pobreza dos indicadores: Uma abordagem geográfica das desigualdades sociais em saúde. Em: Christovam Barcellos, ed. A geografia e o contexto dos problemas de saúde. Rio de Janeiro: Abrasco; 2008. Pp. 107-39.

Manuscrito recebido em 11 de abril de 2012. Aceito em versão revisada em 31 dezembro de 2012. 
ABSTRACT Objective. To review the literature to determine the existence of associations between socioeconomic factors (individual and collective) and the presence of tuberculosis.

Tuberculosis and Methods. A systematic literature review was carried out in SciELO, LILACS, Medsocioeconomic indicators: systematic review of the literature line, and Scopus using the following search terms: poverty, social indicators, socioeconomic factors, and tuberculosis (in Portuguese, English, and Spanish). Studies having individuals as the unit of analysis were classified according to study design and dependent variable. Ecological studies were classified according to levels of spatial aggregation of data and dependent variable. For each article, the following were recorded: study title, country of origin, year the study was carried out, authors, language, objective, level of spatial aggregation, and indicators used in the analysis. Results. For individual level studies, a direct statistical association was observed between tuberculosis and alcohol addiction, HIV coinfection, low schooling, marital status, low income, lack of food, immigration, and previous contact with tuberculosis patients. For collective analyses, an indirect association was observed for variables relating to gross domestic product per capita, human development index, and basic sanitation at the country level. Indicators relating to crowding, poverty density, schooling, decline in family income, and households receiving governmental cash support were directly associated with tuberculosis at different levels of spatial aggregation.

Conclusions. The studies analyzed indicate a persisting relationship between socioeconomic indicators and the production of tuberculosis both at the individual and collective levels. The association between tuberculosis and socioeconomic indicators seems to be influenced by both the level of spatial aggregation and specific characteristics of geographic areas.

Key words Tuberculosis; socioeconomic factors; poverty; review. 